\title{
AVALIAÇÃO da CINÉTICA DE SECAGEM EM ANÁLISE TERMOGRAVIMÉTRICA DO BAGAÇO DE LARANJA
}

\author{
A. ZANETTI ${ }^{1}$, L. C. BENEVIDES ${ }^{2}$, T. P. XAVIER ${ }^{1}$ e T. S. LIRA $^{1,2}$ \\ ${ }^{1}$ Universidade Federal do Espírito Santo, Departamento de Engenharias e Tecnologia \\ ${ }^{2}$ Universidade Federal do Espírito Santo, Programa de Pós-graduação em Energia \\ E-mail para contato: taisa.lira@ufes.br
}

\begin{abstract}
RESUMO - O Brasil é responsável por metade da produção mundial de suco de laranja sendo que em torno de 50\% dessa produção são rejeitadas. Os rejeitos podem ser utilizados como biomassa para o processo de pirólise com a finalidade de obtenção de um conteúdo mais energético. A secagem é primordial para a pirólise porque reduz a umidade presente nos produtos desse processo e permite obtê-los com maior qualidade. Este trabalho tem como objetivo estudar a cinética de secagem do bagaço da laranja por meio da termogravimetria a partir de modelos semi-empíricos em suas formas não isotérmicas. Pôde-se obter a energia de ativação, estimar os parâmetros dos modelos e a partir desses dados observouse que o modelo de Overhults é o que melhor representa a cinética de secagem do bagaço de laranja.
\end{abstract}

\section{INTRODUÇÃO}

A citricultura brasileira provê $50 \%$ da fabricação mundial de suco de laranja e por isso há uma grande geração de resíduos sólidos, como o bagaço, que corresponde a 50\% da massa total da fruta (Farinas et al, 2008). Esse resíduo pode ser aproveitado na forma de biomassa para a conversão de energia limpa. Existem três principais processos termoquímicos de conversão de biomassa: a gaseificação, a combustão e a pirólise (McKendry, 2002). A pirólise é um processo que consiste na degradação térmica da biomassa na ausência total de um agente oxidante ou em quantidades mínimas de modo que a gaseificação não ocorra. A temperatura pirolitíca pode variar de $400{ }^{\circ} \mathrm{C}$ a $800{ }^{\circ} \mathrm{C}$ e os principais subprodutos formados são líquidos (bio-óleo), sólidos (carvão) e gasosos (Kimura, 2009).

A pirólise ocorre em duas etapas: a secagem e a devolatilização. A primeira corresponde à evaporação da água presente na biomassa por meio de transferência de calor e massa, e já a segunda corresponde à degradação térmica dos demais componentes como a celulose, hemicelulose e lignina (Cai e Liu, 2007). A água está presente de forma significativa na biomassa, principalmente no bagaço de laranja (cerca de $85 \%$ em b.u.) (Fiorentin et al., 2012), e por isso a cinética da etapa de secagem deve considerada e avaliada (Chen et al., 2009). Além disso, essa etapa é primordial para a pirólise porque elimina ou reduz a umidade presente nos produtos desse processo influenciando diretamente em sua qualidade.

Dessa forma, este trabalho apresenta como finalidade o estudo da cinética de secagem por meio da análise termogravimétrica (TGA) do bagaço de laranja. Para isso, o 
comportamento da secagem foi avaliado pela variação das taxas de aquecimento e pela aplicação do modelo teórico e os modelos semi-empíricos em suas formas não isotérmicas.

\section{METODOLOGIA}

\subsection{Preparação das amostras do bagaço de laranja}

A biomassa empregada para o estudo da cinética de secagem em análise termogravimétrica foi o bagaço de laranja pera, (constituído de casca, sementes e polpa). $\mathrm{O}$ bagaço foi previamente seco em estufa por 24 horas a $105 \pm 3^{\circ} \mathrm{C}$ e moído.

\subsection{Secagem por análise termogravimétrica}

A partir da amostra preparada realizaram-se experimentos de termogravimetria (TGA) que consiste na técnica na qual a variação de massa de uma amostra é medida em função da temperatura ou do tempo. O equipamento de TGA foi mantido sob fluxo contínuo de atmosfera inerte de nitrogênio à taxa de $50 \mathrm{ml} / \mathrm{min}$. Utilizou-se $15 \mathrm{mg}$ de amostra da biomassa para cada análise e os ensaios dinâmicos foram conduzidos com taxas de aquecimento de 5, 10,20 e $30 \mathrm{~K} / \mathrm{min}$, da temperatura ambiente até a temperatura aproximada de $900 \mathrm{~K}$.

\subsection{Modelos semi-empíricos de cinética de secagem}

A cinética de secagem por análise termogravimétrica foi determinada utilizando-se os modelos semi-empíricos empíricos de Lewis (1921), Brooker et al. (1974), Overhultz et al. (1973) e o modelo teórico de Fick (BROOKER et al., 1992) considerando-se as partículas esféricas, apresentados na Tabela 1. Os dados experimentais foram ajustados às equações de cinética de secagem por meio de regressões não lineares e os parâmetros foram estimados pela minimização dos quadrados dos resíduos. O modelo que apresentasse o maior coeficiente de determinação, $R^{2}$, foi considerado aquele com melhor ajuste.

Tabela 1 - Equações dos modelos de secagem.

\begin{tabular}{cc}
\hline Modelo & \multicolumn{1}{c}{ Equação } \\
\hline Lewis & $M R=\exp \left(-a \exp \left(-\frac{b}{T_{f}}\right) t\right)$ \\
Brooker & $M R=C \exp \left(-a \exp \left(-\frac{b}{T_{f}}\right) t\right)$ \\
Overhultz & $M R=\exp \left[-\left(\exp \left(a+\frac{b}{T_{f}}\right) t\right)^{c}\right]$ \\
Fick & $M R=\frac{6}{\pi^{2}} \sum_{n=1}^{\infty} \frac{1}{n^{2}} \exp \left(\frac{-n^{2} \pi^{2} D_{e f} t}{R^{2}}\right)$ \\
\hline
\end{tabular}

Sendo $T_{f}$ a temperatura do ar, $t$ o tempo e $R$ o raio da partícula. $a, b$ e $c$ são adimensionais e $D_{e f}$ é a difusividade efetiva, a serem estimados. MR é a razão adimensional de umidade da biomassa expressa na Equação 5 simplificada pela desconsideração do termo que expressa o conteúdo de umidade no equilíbrio. $M$ é o teor de umidade na temperatura $T$ e $M_{0}$ a umidade inicial, sempre em base seca (Chen et al., 2013). 


$$
\mathrm{MR}=\frac{\mathrm{M}}{\mathrm{M}_{0}}
$$

\section{RESULTADOS E DISCUSSÕES}

A Figura 1a e $1 \mathrm{~b}$ representam as curvas referentes à perda de massa (TG) e à taxa da perda de massa (DTG) respectivamente, nos ensaios dinâmicos para a amostra de bagaço de laranja. Estas curvas apresentaram deslocamento gradual para maiores temperaturas com o aumento da taxa de aquecimento. Os resultados de TG e DTG com taxa de aquecimento de $30 \mathrm{~K} / \mathrm{min}$ para o bagaço de laranja são apresentados na Figura 2.

Figura 1 - Curva experimental (a) da perda de massa percentual (TG) e (b) da taxa da perda de massa (DTG) em função da temperatura em diferentes taxas de aquecimento para o bagaço de laranja.

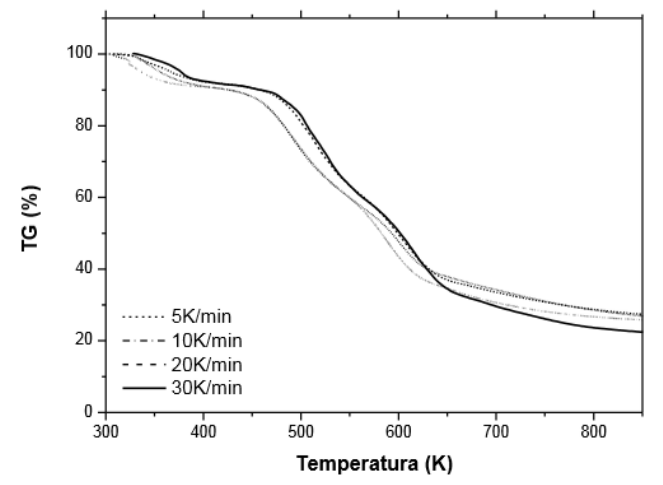

(a)

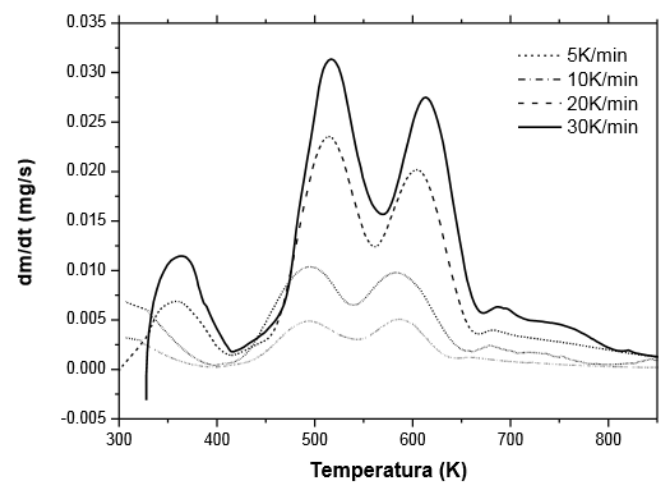

(b)

Figura 2 - TG e DTG do ensaio do bagaço de laranja a uma taxa de aquecimento de 30K/min.

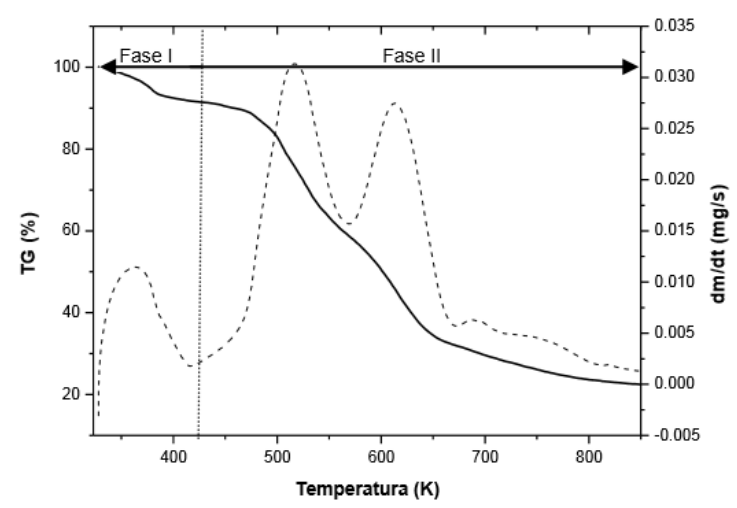

A partir da Figura 2 é possível observar a secagem (Fase I) e a devolatização (Fase II). Na primeira, a distribuição de água na biomassa dá-se de duas formas, pela água livre e a água "ligada". A interação da água livre com a biomassa é fraca e sua evaporação ocorre a uma temperatura mais baixa do que a da água ligada, cuja interação com a biomassa é mais forte. Aos $373 \mathrm{~K}$ a água livre já foi completamente evaporada e a partir dessa temperatura inicia-se a evaporação da água ligada até aproximadamente $423 \mathrm{~K}$ (Chen et al., 2013).

As Figuras 3a e 3b mostram as curvas de secagem do bagaço de laranja em função da temperatura e do tempo, respectivamente, a taxas de aquecimento de $5,10,20$ e $30 \mathrm{~K} / \mathrm{min}$. 
Observa-se que houve uma redução da umidade do material com a diminuição da taxa de aquecimento e os aumentos da temperatura e do tempo de secagem.

Figura 3 - Curva de secagem do bagaço de laranja em função da (a) temperatura e do (b) tempo em relação a diferentes taxas de aquecimento.

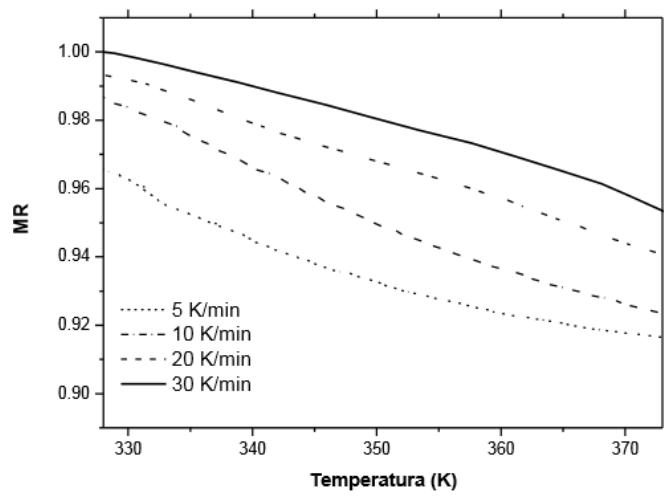

(a)

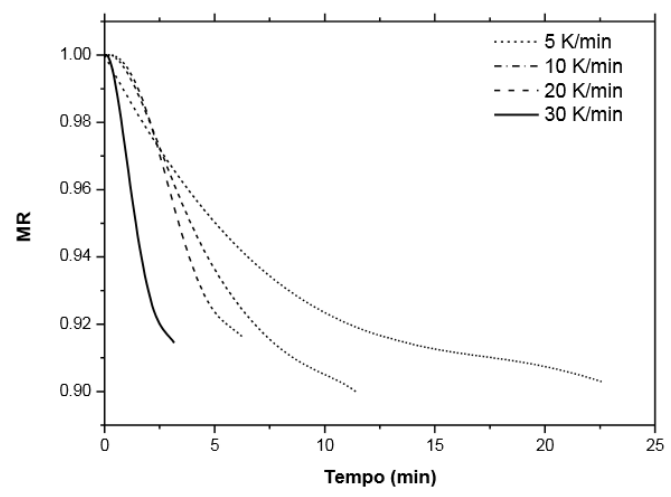

(b)

Os parâmetros cinéticos foram determinados para a fase de perda de água livre, no qual há variação da temperatura ambiente até $100^{\circ} \mathrm{C}$ para os ensaios dinâmicos a cada taxa de aquecimento estudada. Os resultados encontram-se na Tabela 2. Observou-se que os valores de energia de ativação para os modelos mencionados ficaram na faixa de 2,198 e 19,242 $\mathrm{KJ} / \mathrm{mol}$, resultado similar com o reportado na literatura para secagem de resíduos agroindustriais, palha de milho e de trigo, $5-30 \mathrm{KJ} / \mathrm{mol}$ (Chen et al., 2013). A Figura 4 apresenta as curvas de secagem experimentais e as estimadas pelos modelos da Tabela 1.

Tabela 2 - Parâmetros de cinética de secagem por meio de termogravimetria do bagaço de laranja.

\begin{tabular}{|c|c|c|c|c|c|c|}
\hline \multirow[t]{2}{*}{ Modelo } & \multirow[t]{2}{*}{ Taxa de Aquecimento $\left(\mathrm{K} \min ^{-1}\right)$} & \multicolumn{3}{|c|}{ Parâmetros } & \multirow{2}{*}{$\frac{\text { Ea }}{\left(\mathrm{KJ} \mathrm{mol}^{-1}\right)}$} & \multirow[t]{2}{*}{$\mathbf{R}^{2}$} \\
\hline & & $a$ & $b$ & $c$ & & \\
\hline \multirow[t]{4}{*}{ Lewis } & 5 & 0,0329 & 529,960 & - & 4,406 & 0,9954 \\
\hline & 10 & 0,0571 & 541,063 & - & 4,498 & 0,9907 \\
\hline & 20 & 6,424 & 2213,286 & - & 18,401 & 0,9942 \\
\hline & 30 & 18,171 & 2314,415 & - & 19,242 & 0,9959 \\
\hline \multirow[t]{4}{*}{ Brooker } & 5 & 0,012 & 144,149 & 1,005 & 2,198 & 0,9973 \\
\hline & 10 & 0,006 & 309,874 & 1,011 & 2,576 & 0,9977 \\
\hline & 20 & 0,871 & 1447,917 & 1,005 & 12,030 & 0,9979 \\
\hline & 30 & 1,995 & 1470,264 & 1,003 & 12,223 & 0,9990 \\
\hline \multirow[t]{5}{*}{ Overhultz } & 5 & $-8,687$ & 1457,359 & 1,083 & 12,116 & 0,9999 \\
\hline & 10 & $-7,479$ & 1707,459 & 2,409 & 14,195 & 0,9999 \\
\hline & 20 & $-4,809$ & 964,838 & 3,129 & 8,021 & 0,9995 \\
\hline & 30 & $-4,870$ & 1280,578 & 2,632 & 10,647 & 0,9994 \\
\hline & & \multicolumn{4}{|c|}{$D_{\text {ef }}\left(m^{2} s^{-1}\right)$} & $\mathbf{R}^{2}$ \\
\hline \multirow[t]{4}{*}{ Fick } & 5 & \multicolumn{4}{|c|}{$3,013 \times 10^{-12}$} & 0,6330 \\
\hline & 10 & \multicolumn{4}{|c|}{$3,383 \times 10^{-12}$} & 0,2931 \\
\hline & 20 & \multicolumn{4}{|c|}{$2,113 \times 10^{-12}$} & 0,1647 \\
\hline & $30 *$ & \multicolumn{4}{|c|}{-} & - \\
\hline
\end{tabular}

* não houve ajuste. 
Figura 4 - Comparação da umidade relativa obtida experimentalmente e pelos diferentes modelos em função do tempo para diferentes taxas de aquecimento: (a) $5 \mathrm{~K} / \mathrm{min}$, (b) $10 \mathrm{~K} / \mathrm{min}$, (c) $20 \mathrm{~K} / \mathrm{min} \mathrm{e} \mathrm{(d)} 30 \mathrm{~K} / \mathrm{min}$.

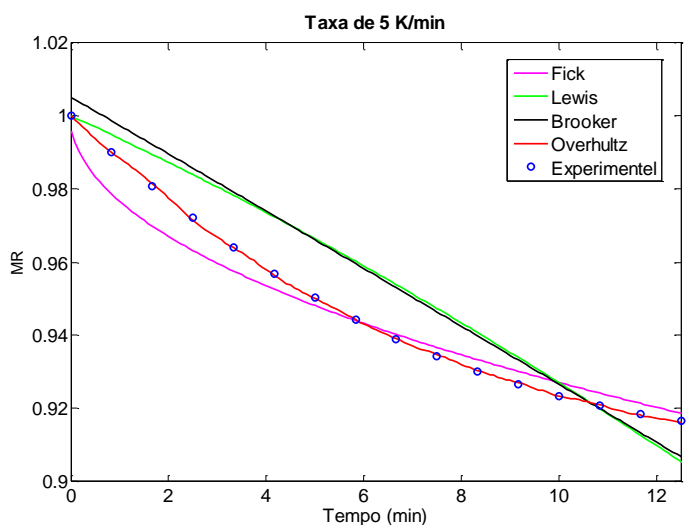

(a)

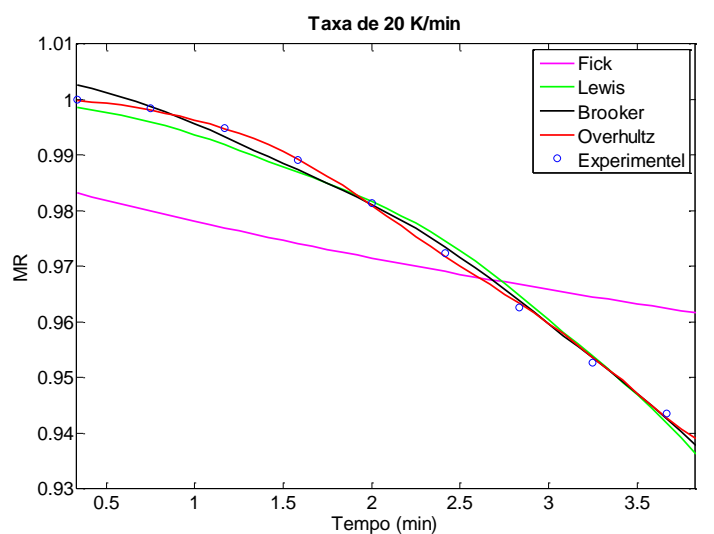

(c)

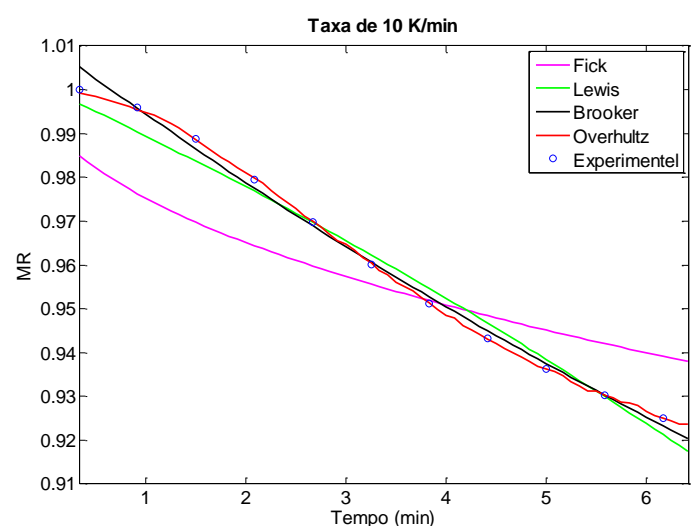

(b)

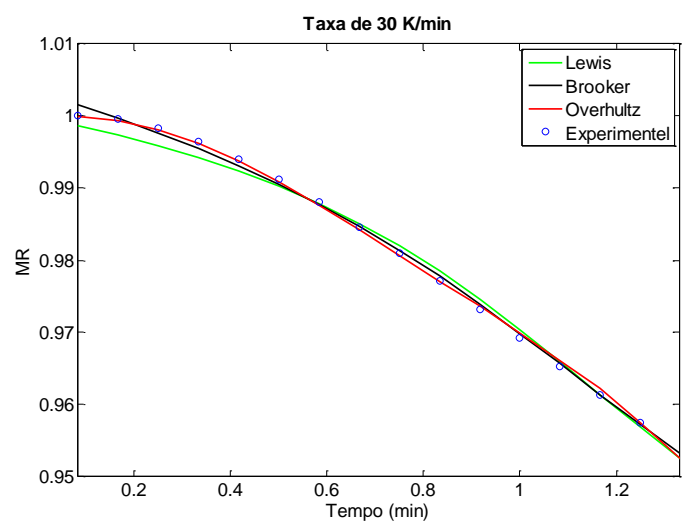

(d)

Pela análise das curvas de secagem e pelos coeficientes de determinação, o modelo de Overhultz obteve os melhores ajustes para a cinética de secagem, cujo $\mathrm{R}^{2}$ foi superior a 0,9994. A média dos valores de energia de ativação para este modelo é 11,245 KJ/mol. Podese observar que o modelo de Fick apresentou o pior ajuste dos dados experimentais com $\mathrm{R}^{2}<0,6330$, sendo que esse valor decresceu com o aumento da taxa de aquecimento e que não foi possível o ajuste na taxa de $30 \mathrm{~K} / \mathrm{min}$. A difusividade efetiva média variou de $3,013 \times 10^{-12}$ até $2,113 \times 10^{-12} \mathrm{~m}^{2} \cdot \mathrm{s}^{-1}$ para as taxas de aquecimento entre 5 e $20 \mathrm{~K} / \mathrm{min}$, respectivamente. Possivelmente, a falta de adequação do último modelo deve-se a consideração da difusividade como um parâmetro constante para obtenção da solução analítica da $2^{\mathrm{a}}$ Lei da Fick, sendo que ela varia com a temperatura que não é constante nos experimentos.

\section{CONCLUSÕES}

A partir do estudo realizado foi possível avaliar o processo de secagem não isotérmica do bagaço da laranja pela termogravimetria a diferentes taxas de aquecimento. Na medida em que ocorreu o aumento da temperatura e a redução da taxa de aquecimento houve decréscimo da umidade da biomassa. Determinou-se também que a energia de ativação para os modelos semiempíricos de secagem empregados estão na faixa de 2,198 e 19,242 KJ/mol. Além disso, pode-se 
concluir que o modelo de Overhults é o que melhor se adequa a cinética de secagem do bagaço de laranja já que possui o maior valor de $\mathrm{R}^{2}$.

\section{REFERÊNCIAS}

BROOKER, D.B., BAKKER-ARKEMA, F.W., HALL, C.W. Drying cereal grains. The Avi Publish Company, Inc., Westport, 1974.

BROOKER, D. B.; BAKKER-ARKEMA, F. W.; HALL, C. W. Drying and storage of grains and oilseeds. New York: The AVI Van Nostrand Reinhold, 1992. 450 p.

CAI, J.M., LIU, R.H. Research on water evaporation in the process of biomass pyrolysis. Energy Fuels, v. 21, p. 3695-3697, 2007.

CHEN, L.; XING, L.; HAN, L. Renewable energy from agro-residues in China: Solid biofuels and biomass briquetting technology. Renewable and Sustainable Energy Reviews, v. 13, p- 2689-2695, 2009.

CHEN, D, ZHENG, Y, ZHU, X. In-depth investigation on the pyrolysis kinetics of raw biomass, Part I: Kinetic analysis for the drying and devolatilization stages. Bioresource Technology, v. 131, p. 40-46, China, 2013.

FARINAS, C. S., LEMO, V., ZÚNIGA, U. F., BERTUCI NETO, V., COURI, S. Avaliação de Diferentes Resíduos Agroindustriais como Substratos para a Produção de Celulases por Fermentação Semi-sólida. Embrapa Instrumentação Agropecuária, São Carlos, SP, 2008.

FIORENTIN, L. D.; MENON B. T.; ALVES J. A., BARROS, S. T. D. de; PEREIRA, N. C.; MOTTA LIMA, O. C. da; MODENES, A. N. Análise de secagem do bagaço de laranja em camada fina utilizando modelos semi-teóricos e empíricos. Revista Engevista, v. 14, n. 1, p. 22-23, 2012.

KIMURA, L. M. Uma contribuição à pirólise de biomassa: avaliação de alguns resíduos da agroindústria como fonte de energia alternativa. 2009. 105 f. Dissertação (Mestrado)Curso de Engenharia Química, Universidade Federal de Uberlândia, Uberlândia, Minas Gerais, 2009.

LEWIS, W.K. The rate of drying of solids materials. Ind. Eng. Chem. vol 13, p. 427, 1921.

McKENDRY, P. Energy production from biomass (part 2): conversion technologies. Revista Elsevier Science. v. 83, p. 47-54, 2002.

OVERHULTZ, D.G.; WHITE, G.M.; HAMILTON, H.E. et al. Drying soybeans with heated air. Transactions of the ASAE, p. 112-113, 1973. 